\title{
Modélisation du transport littoral
}

\author{
Catherine Villaret \\ Laboratoire National d'Hydraulique et Environnement, \\ Electricité De France, $R \& D$, \\ 6 Quai Watier, 78400 Chatou France. \\ E-mail: catherine.villaret@edf.fr
}

\section{Résumé:}

L'effet de la houle sur la dynamique sédimentaire doit être pris en compte par une modélisation intégrée des forçages hydrodynamiques (houle, courants de houle et de marée) et du transport sédimentaire. Différents modèles du système hydro-informatique Telemac ont été mis en œuvre pour calculer le transport littoral sur un cas test schématique (plage rectiligne), puis sur un cas réel (plage macro-tidale, située à l'Est de Dunkerque). Nous présentons ici la méthode de couplage, et discutons la sensibilité des résultats aux lois de frottement et aux formules de transport.

\section{Abstract:}

In coastal areas, the effect of waves on sand transport rates needs to be accounted for by an integrated modeling of hydrodynamic forcing terms (waves, wave-induced and tidal currents) and resulting sand transport rates. The hydro-informatic system Telemac is applied here on two tests cases: 1) a straight coast line, where the littoral transport is induced by an incident wave, and 2) a macro-tidal beach located at the East of Dunkerque. We discuss here the general method and the sensitivity of the results on bottom friction laws and sand transport formulae.

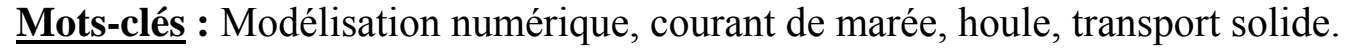

\section{Introduction}

En zone côtière, la houle et les courants jouent des rôles complémentaires sur la dynamique sédimentaire : la houle a pour effet de favoriser la mise en suspension des sédiments qui sont ensuite transportés par les courants moyens. La variation spatiale des taux de transport entraîne des phénomènes d'érosion ou d'accrétion, avec des conséquences importantes pour la gestion du littoral. Il est nécessaire de construire des outils de modélisation permettant de prédire précisément les taux de transport et les évolutions morphodynamiques qui en résultent.

Le calcul des forçages hydrodynamiques nécessite la mise en œuvre d'un code de propagation de houle et d'un modèle courantologique afin de calculer les courants de marée ainsi que les courants de houle. La méthodologie utilisée dépend des conditions rencontrées (houle dominante ou marée dominante), et consiste à enchaîner plusieurs calculs, afin de tenir compte des interactions entre la houle et les courants.

Sont présentées ici deux applications : la première concerne le calcul du transport littoral sous l'effet d'une houle oblique, le long d'une plage rectiligne et uniforme, et la seconde, le calcul du transport littoral sur une plage macro-tidale, située à l'Est de Dunkerque.

Après une présentation rapide des codes de calcul et de la méthode de couplage utilisée, nous discutons la sensibilité des résultats aux lois de frottement et aux formules de transport. 


\section{Système Telemac}

Nous avons utilisé plusieurs modèles du système hydro-informatique Telemac, développé au LNHE (cf. [6]):

-le code hydrodynamique Telemac-2d, qui résout les équations de St-Venant avec prise en compte éventuelle de termes de forçage dus à la houle (contraintes de radiation) ou à la marée,

-le code de propagation de houle Tomawac, modèle de troisième génération, qui prend en compte les effets de la réfraction et de la dissipation d'énergie dans la zone de déferlement,

-le code morphodynamique Sisyphe (co-propriété du Cetmef, UTC, Edf et Sogreah), qui calcule le taux de transport en fonction du forçage hydrodynamique et résout une équation pour l'évolution du fond.

La méthode de couplage consiste à enchaîner plusieurs calculs, en échangeant les fichiers de résultats entre les codes. Un premier calcul de marée est réalisé à l'aide de Telemac-2d. Le calcul de la houle est réalisé ensuite en utilisant le code Tomawac en régime non-permanent, afin de tenir compte des variations de la hauteur d'eau au cours de la marée et de la réfraction des vagues par les courants. Le calcul courantologique (Telemac-2d) est ensuite réitéré en tenant compte des contraintes de radiation calculées par le modèle de houle. En pratique, cette méthode générale pourra être simplifiée, en régime dominé par la houle ( $\mathrm{cf} \S 4$ ) ou par la marée (cf §5).

Le code morphodynamique Sisyphe utilise les résultats des calculs hydrodynamiques préalables (houle et courant). La prise en compte de l'effet de la houle sur le transport sédimentaire a fait l'objet de développements récents, détaillés au $§ 3$.

\section{$\underline{\text { 3.Prise en compte des effets de la houle }}$}

Une houle linéaire et symétrique ne produit pas de transport net, mais favorise la mise en suspension des sédiments. Cet effet est dû aux cisaillements importants, créés dans la couche limite par l'écoulement oscillatoire. Le frottement est donc un paramètre essentiel, et son calcul est intimement lié au paramètre de rugosité qui dépend lui-même de la nature du fond. En dehors de la zone de déferlement, la houle est aussi responsable de la formation de rides, qui modifient la structure de la couche limite, avec des conséquences importantes sur les mécanismes de transport.

Nous détaillons ici les développements réalisés dans le logiciel Sisyphe, pour prendre en compte les effets de la houle sur le transport solide. Ces développements, détaillés dans le Manuel Utilisateur [12], ont porté sur les points suivants :

(1) le calcul de la contrainte de frottement, en tenant compte des interactions entre la houle et le courant,

(2) la prise en compte des rides générées par la houle,

(3) la programmation de formules de transport solide adaptées.

\section{Calcul du frottement}

On calcule dans un premier temps la contrainte de frottement due à la houle, en fonction $\mathrm{du}$ courant orbital sur le fond $\mathrm{U}_{0}$, par le biais d'un coefficient de frottement, noté $f_{\mathrm{w}}$. En régime turbulent rugueux, $\mathrm{f}_{\mathrm{w}}$ est calculé par la formule de Swart [9], en fonction du rapport $\mathrm{A}_{0} / \mathrm{k}_{\mathrm{s}}, \mathrm{k}_{\mathrm{s}}$ étant le coefficient de rugosité, $\mathrm{A}_{0}=\mathrm{U}_{0} / \omega$, l'amplitude du mouvement orbital, et $\omega$ la pulsation.

L'interaction non-linéaire entre la houle et le courant se traduit par une augmentation des contraintes de frottement, moyenne et maximale. La méthode de Soulsby ([8], p.183) qui consiste à paramétrer les résultats obtenus par différents modèles de turbulence, a été programmée. 


\section{Calcul des dimensions des rides}

La méthode de Wiberg et Harris [13] a été programmée pour calculer les dimensions des rides à l'équilibre. La formulation retenue, décrite dans le rapport [4], tient compte aussi des effets du courant. Le coefficient de rugosité est pris égal à la hauteur des rides $\left(k_{\mathrm{s}}={ }_{\mathrm{r}}\right)$, en accord avec la formule de Bijker [3].

\section{Formules de transport}

Un certain nombre de formules de la littérature qui permettent de prendre en compte l'effet d'augmentation du taux de transport par la houle, ont été programmées. Nous avons utilisé dans les applications la formule in-stationnaire de Dibajnia et Watanabe ('DW') [5], la formule quasi-stationnaire de Bijker et la formule énergétique de Bailard [2]. Certaines formules ont été adaptées afin de faciliter leur utilisation et/ou d'améliorer leur performance. Par exemple, le coefficient empirique (noté b) de la formule de Bijker varie de $b=2$ à l'extérieur de la zone de déferlement, à $b=5$ l'intérieur (cf. [10]). Pour les formules de Bailard et de 'DW', la houle est supposée sinusoïdale, ce qui permet d'expliciter les termes instationnaires. Les formulations, telles qu'elles ont été programmées dans Sisyphe, sont détaillées dans les rapports [11] et [12].

\section{Transport littoral le long d'une plage rectiligne}

\section{Présentation du cas test}

La première application concerne le cas d'une plage rectiligne et uniforme soumise à l'action d'une houle seule (sans marée). Au large, la houle se propage avec un angle d'incidence de $45^{\circ}$ par rapport à la plage. La période de pic imposée aux limites est de $8 \mathrm{~s}$, pour une hauteur significative de $1 \mathrm{~m}$. La plage, en pente rectiligne et uniforme de $5 \%$, est recouverte d'un sable fin d'un diamètre moyen $\mathrm{D}_{50}=300 \mu \mathrm{m}$. La hauteur d'eau est de $10 \mathrm{~m}$ au large. Le domaine, de $1000 \mathrm{~m}$ de longueur sur $200 \mathrm{~m}$ de large, est discrétisé en éléments triangulaires de $5 \mathrm{~m}$ x $20 \mathrm{~m}$, soit un total 2091 nœuds. L'axe Oy est dirigé dans le profil de plage, la plage étant située en $\mathrm{y}=200 \mathrm{~m}$.

\section{Calculs hydrodynamiques}

Un premier calcul a été réalisé avec le code de propagation de la houle. Le long des frontières latérales, on impose des conditions de périodicité qui consistent à recopier à chaque instant les résultats obtenus au milieu du domaine. Les résultats sont ainsi parfaitement uniformes dans la direction parallèle à la plage.

$\mathbf{H}_{\mathbf{s}}(\mathbf{m})$

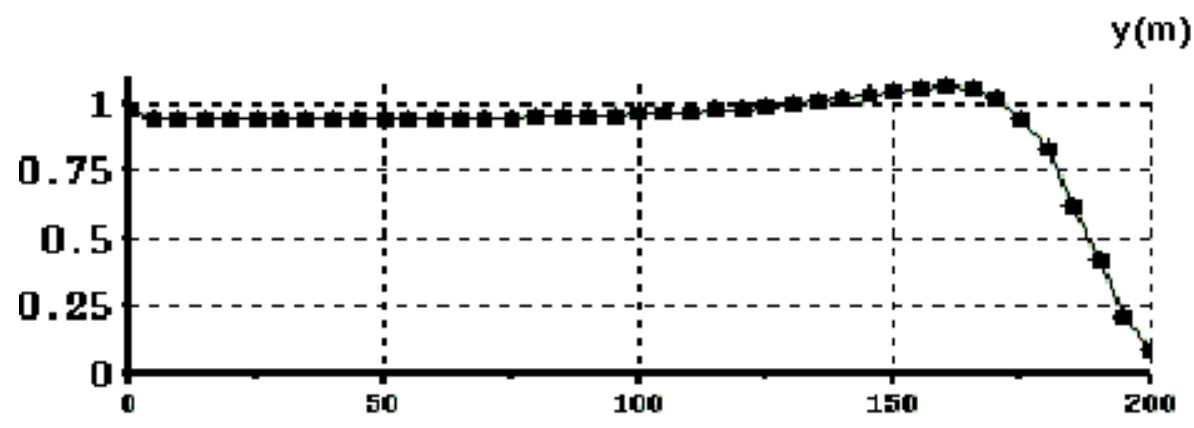

Figure 1: Variation de la hauteur de houle dans le profil de plage (Tomawac - houle incidente : $T_{p}=8 \mathrm{~s}, H_{s}=1 \mathrm{~m}, h=10 \mathrm{~m}, \quad=45^{\circ}$ ) 
La figure1 montre la variation dans le profil de plage de la hauteur de houle : la houle s'amplifie aux abords de la côte par réfraction, pour s'atténuer à cause de la dissipation d'énergie dans la zone de déferlement (cf. [1]). Les effets de la réfraction se manifestent aussi par une rotation de la houle, celle-ci s'orientant progressivement dans une direction normale à la côte. La zone de déferlement s'étend sur une largeur de $40 \mathrm{~m}$. Le point de déferlement est situé à une profondeur de $2 \mathrm{~m}$; la hauteur maximale de la houle est alors de $1.06 \mathrm{~m}$.

Le code courantologique tient compte des termes de forçage par la houle (ou contraintes de radiation), afin de calculer le courant littoral, ainsi que les variations du plan d'eau. La difficulté principale est alors d'imposer de manière cohérente les conditions aux limites. La méthode retenue consiste à imposer des conditions de périodicité sur les frontières ouvertes (droite et gauche), sur les vitesses, en laissant les hauteurs d'eau libres.

Les résultats du modèle courantologique, illustrés sur les figures 2.a et 2.b, ont été obtenus pour un coefficient de rugosité constant et uniforme: $\mathrm{k}_{\mathrm{s}}=3 \mathrm{D}_{50}$, soit $\mathrm{k}_{\mathrm{s}}=0.9 \mathrm{~mm}$, ce qui correspond à un coefficient de Chézy de 79. Le courant littoral, dirigé parallèlement à la côte, est confiné à l'intérieur de la zone de déferlement, où il atteint une intensité maximale de 1.89 $\mathrm{m} / \mathrm{s}$. Ces résultats sont en accord avec la théorie de Longuet-Higgins (1970) : la valeur calculée par le modèle est cependant inférieure à la valeur théorique de $2.10 \mathrm{~m} / \mathrm{s}$, ce qui est normal puisque la solution analytique ne tient pas compte du frottement latéral.

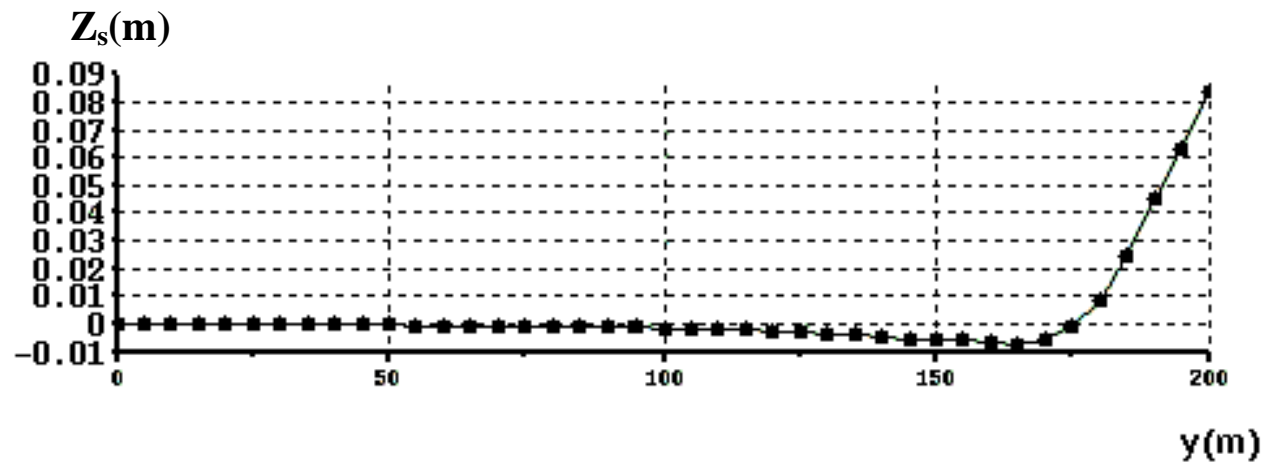

Figure 2a: Variation du niveau moyen (Set-up/Set-down)

$\mathrm{U}(\mathrm{m} / \mathrm{s})$

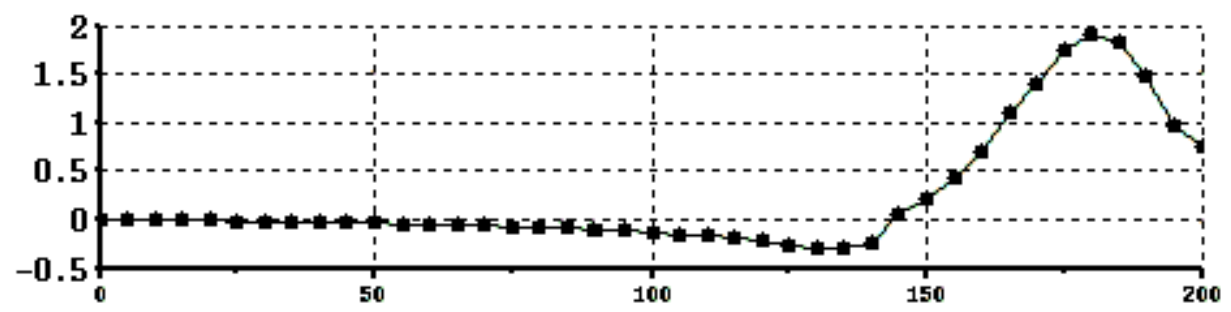

$y(m)$

Figure 2b: Courant littoral

Figure 2: Effet des contraintes de radiation (Telemac2d - Coefficient de frottement : $k_{s}=3 D_{50}, D_{50}=0.3 \mathrm{~mm}$ ) 
La figure 2a permet d'observer les variations du niveau moyen : en dehors de la zone de surf, la surface libre décroît légèrement, pour augmenter dans la zone de déferlement. Le niveau moyen est alors proportionnel au gradient bathymétrique, et le set-up atteint sa valeur maximale au niveau du rivage $(\Delta \mathrm{h}=8.5 \mathrm{~cm})$.

On observe cependant sur la figure 2.b la présence d'un courant de retour parasite, dû probablement à une re-circulation au niveau de la frontière sortante. Ce courant de retour a une intensité maximale de $0.3 \mathrm{~m} / \mathrm{s}$, soit $15 \%$ environ du courant littoral. Son influence sur le transport solide reste cependant négligeable.

\section{$\underline{\text { Transport solide }}$}

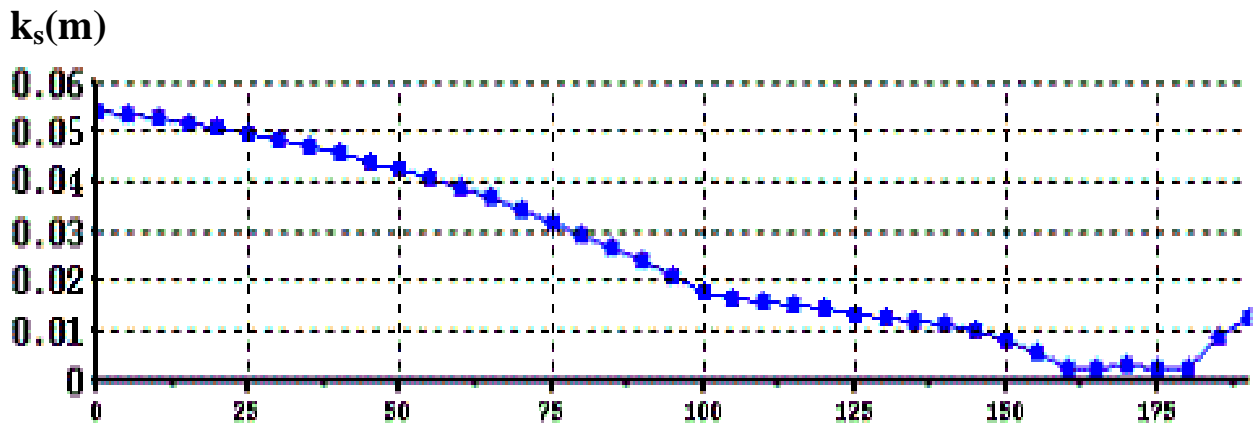

Figure 3a: Variation du coefficient de rugosité sur fond ridé $\left(k_{s}=\eta_{r}\right)$

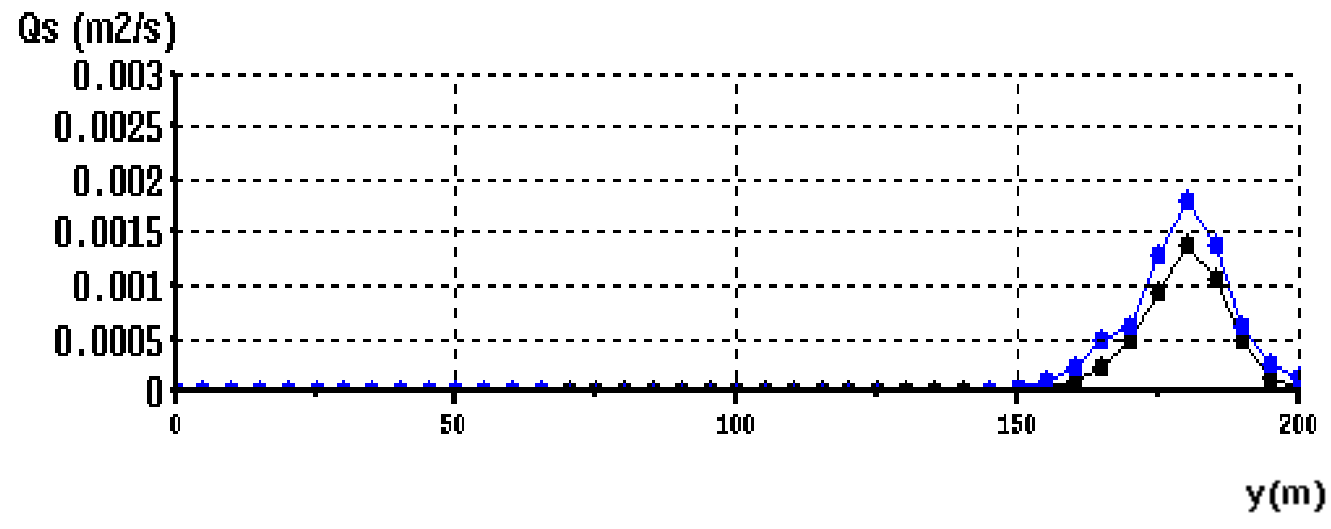

Figure 3b: Influence du coefficient de frottement sur le taux de transport (formule de Bijker - en noir : fond plat - en bleu : fond ridé) 


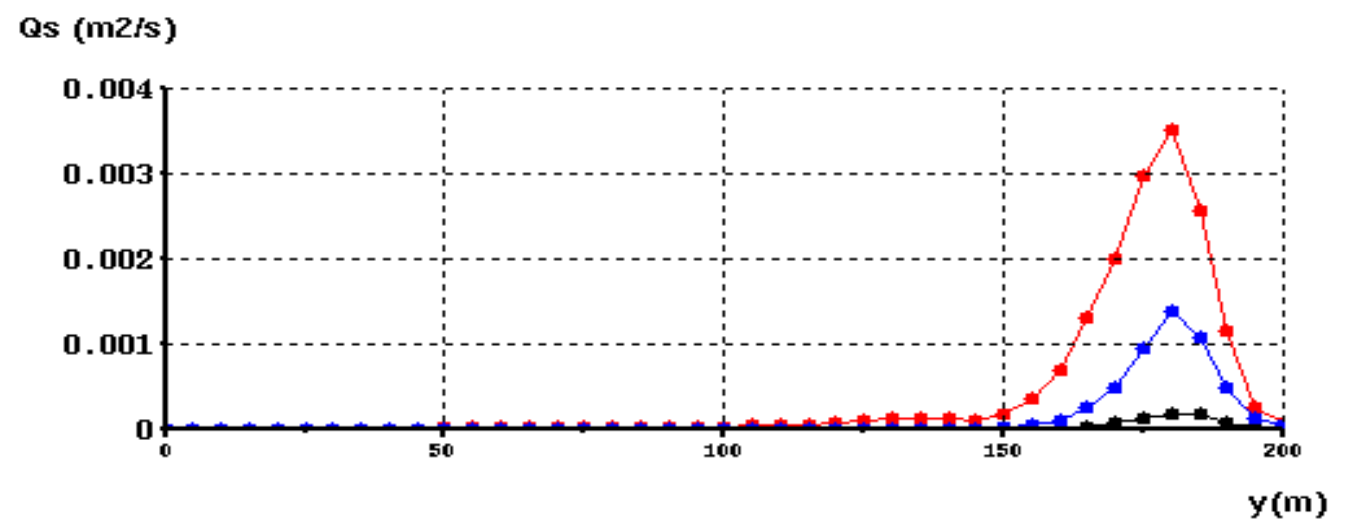

Figure 3c: Intercomparaison des formules de transport solide $\left(k_{s}=3 D_{50}\right)$ (en noir : 'DW' - en bleu : Bijker - en rouge : Bailard)

Figure 3: Calcul du transport solide et des dimensions des rides (Sisyphe : formule de Bijker)

Les résultats du modèle de transport sédimentaire sont illustrés sur la figure 3. La figure 3.a montre les variations du coefficient de rugosité $\left(\mathrm{k}_{\mathrm{s}}=\eta_{\mathrm{r}}\right)$ dans le profil de plage : la présence de rides au large se traduit par une augmentation du coefficient de rugosité, tandis que le transport s'effectue sur fond plat ('sheet flow') dans la zone de déferlement. Les rides se reforment ensuite vers le haut de plage, lorsque l'énergie de la houle a été entièrement dissipée. La figure 3.b montre la variation du taux de transport qui atteint sa valeur maximale à l'intérieur de la zone de déferlement. Pour une même formule (ici, la formule de Bijker), les résultats obtenus sont sensibles au coefficient de frottement, avec une augmentation du taux de transport de l'ordre de $30 \%$ pour un fond ridé, par rapport au cas d'un fond plat. Pour une même loi de frottement, les résultats obtenus sont très sensibles au choix de la formule de transport solide, comme le montre la figure 3.c.

Les résultats numériques, intégrés dans le profil de plage, sont ensuite comparés à des formules globales (cf. [14] et [15]) . La formule du CERC (Table 1.a) surestime le taux de transport par rapport aux résultats du modèle (Table1.b). La formule de Bailard surestime les taux de transport, pour donner des résultats intégrés du même ordre de grandeur que ceux de la formule du CERC. La formule 'DW' sous-estime fortement les taux de transport. On utilisera par la suite la formule de Bijker qui, combinée à une loi de frottement sur fond ridé, donne des résultats 'moyens' (voir aussi [11]). A titre indicatif, on donne aussi la valeur calculée par la formule empirique de Soulsby-Van Rijn (cf. [8]), qui préconise une valeur aberrante du coefficient de rugosité $\left(\mathrm{k}_{\mathrm{s}}=18 \mathrm{~cm}\right)$.

Table 1a. Intercomparaison des formules intégrées

\begin{tabular}{|c|c|c|}
\hline Formules & $\begin{array}{c}\text { CERC } \\
{[14]}\end{array}$ & CERC [15] \\
\hline $\mathrm{Q}_{\mathrm{s}}\left(\mathrm{m}^{3} / \mathrm{s}\right)$ & 0.244 & 0.204 \\
\hline
\end{tabular}


Table $1 \boldsymbol{b}$ Intercomparaison des résultats numériques (Sisyphe)

\begin{tabular}{|c|c|c|c|c|}
\hline $\begin{array}{c}\mathrm{Q}_{\mathrm{s}} \\
\left(\mathrm{m}^{3} / \mathrm{s}\right)\end{array}$ & Bijker & $\begin{array}{c}\text { Soulsb } \\
\mathrm{y}\end{array}$ & Bailard & 'DW' \\
\hline $\mathrm{k}_{\mathrm{s}}=3 \mathrm{D}_{50}$ & 0.025 & - & 0.080 & 0.004 \\
$\mathrm{k}_{\mathrm{s}}=\eta_{\mathrm{r}}$ & 0.036 & - & 0.156 & 0.007 \\
$\mathrm{k}_{\mathrm{s}}=18 \mathrm{~cm}$ & - & 0.224 & - & - \\
\hline
\end{tabular}

\section{Application au littoral Est-Dunkerquois}

La seconde application concerne le calcul du transport littoral le long des plages sableuses du littoral Est-Dunkerquois. Le site retenu est caractérisé par un environnement macro-tidal complexe, avec un estran très étendu et un système de barres et de bâches. Au large, les bancs de Flandre orientés parallèlement à la côte constituent des brise-lames naturels. Cette portion du littoral est fragilisée par une érosion importante, en particulier pendant les événements extrêmes. Le fond est constitué d'un sable relativement fin et uniforme $\left(\mathrm{D}_{50}=300 \mu \mathrm{m}\right)$. L'objectif est ici de calculer le transport solide lors des tempêtes hivernales.

Le modèle hydrodynamique s'étend de Cap Blanc Nez à l'Ouest, jusqu'à Nieuwpoort (Belgique) à l'Est, ce qui représente environ $77 \mathrm{~km}$ de littoral. Au large, le modèle s'étend jusqu'à environ $48 \mathrm{~km}$ des côtes afin d'englober les bancs situés au large. La bathymétrie est illustrée sur la figure 4.a. La taille des mailles est de l'ordre de $200 \mathrm{~m}$ près de la côte et atteint $2 \mathrm{~km}$ au large. Le maillage comporte 10786 nœuds.

$\mathrm{h}(\mathrm{m})$

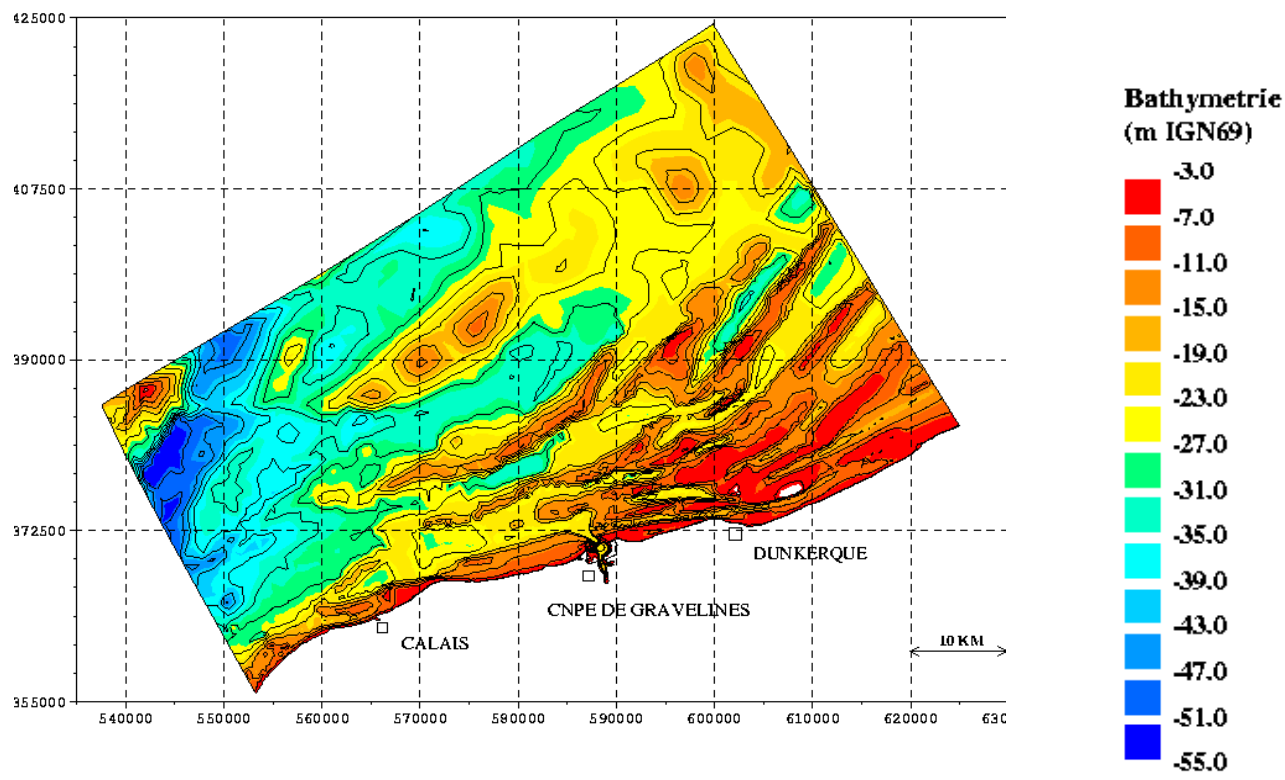

Figure 4a : Littoral Est Dunkerquois : bathymétrie 


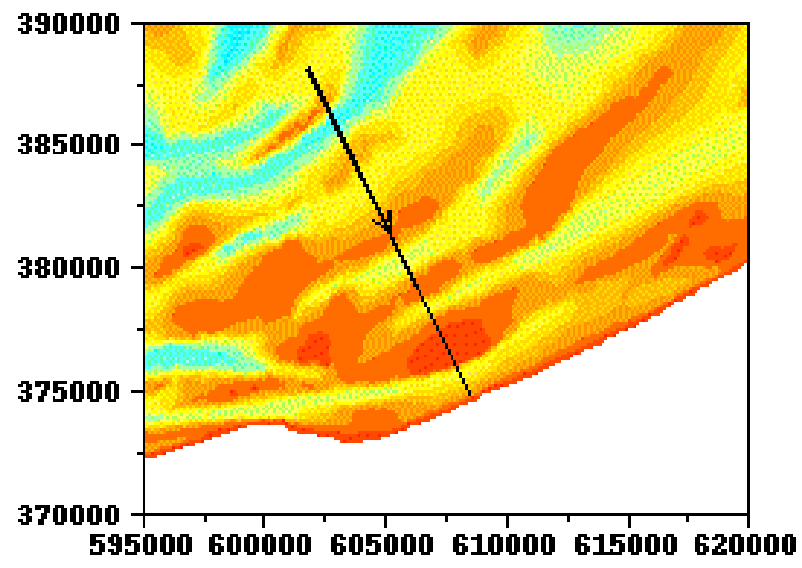

Figure $4 \boldsymbol{b}$ Position du profil

Un premier calcul de marée avec Telemac-2d reproduit des conditions de vive-eau extrême. Des conditions périodiques pour les courants et les hauteurs d'eau sont imposées le long des frontières liquides. La figure 5 illustre le champ des courants à marée haute. Le courant de flot, dominant, porte vers le nord-est, puis s'inverse au cours du jusant pour s'orienter vers le sud-ouest. Au large de Dunkerque, les courants de flot dépassent $1 \mathrm{~m} / \mathrm{s}$.

Le code Tomawac est ensuite utilisé en régime non-permanent. La houle centennale imposée le long des frontières liquides a une hauteur de $7,4 \mathrm{~m}$ pour une période de $8 \mathrm{~s}$. C'est à marée haute que les hauteurs de houle sont maximales à proximité des côtes, comme le montre la Fig. 6.b.

Le code Sisyphe calcule ensuite les variations du transport sédimentaire en réponse aux variations du forçage dû à la houle et aux courants. La figure 6 montre un détail de la zone d'étude, le long d'un profil de plage au niveau de Leffrinckouke (Est de Dunkerque) comme indiqué sur la figure 4.a. Comme pour la houle, c'est aussi à marée haute que les taux de transport sont maximum, comme illustré sur la figure 6.c. Le transport net, intégré sur une période de la marée, est donc dirigé dans le sens des courants de flot, vers le nord-est. Par ailleurs, les résultats mettent en évidence une intensification des taux de transport au niveau des bancs sableux.
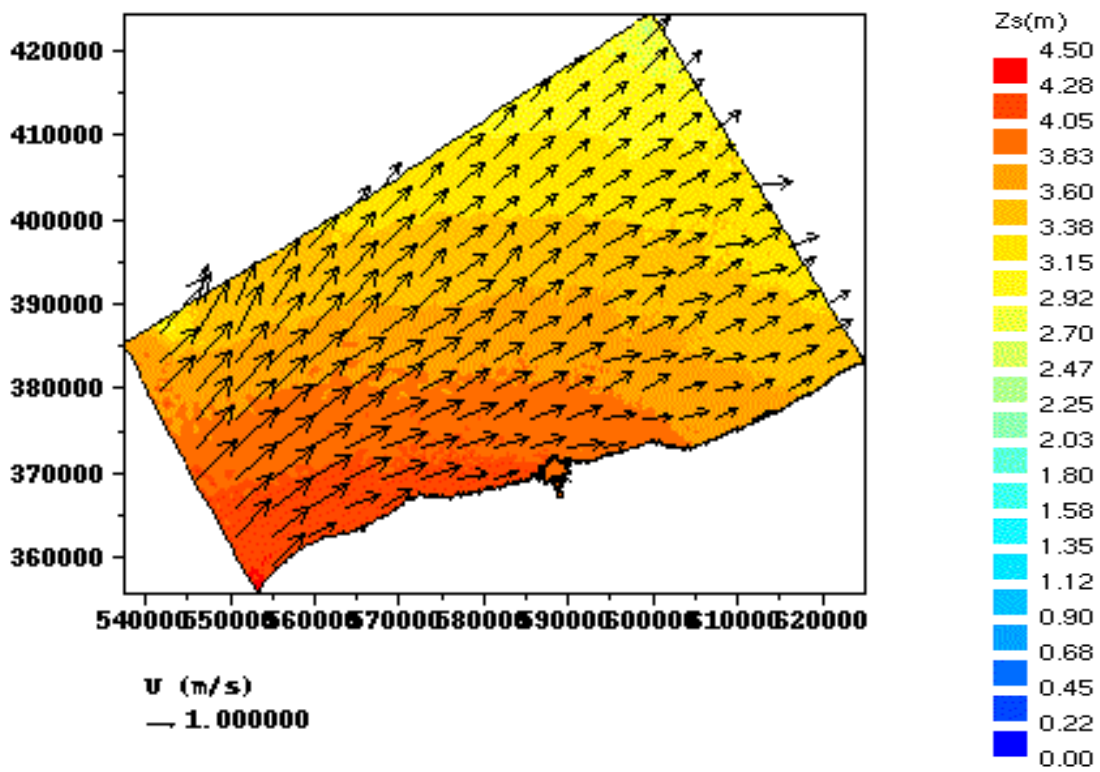

Figure 5: Champ de courant à marée haute et niveau de la surface libre (Telemac-2d : Marée de vive-eau ) 


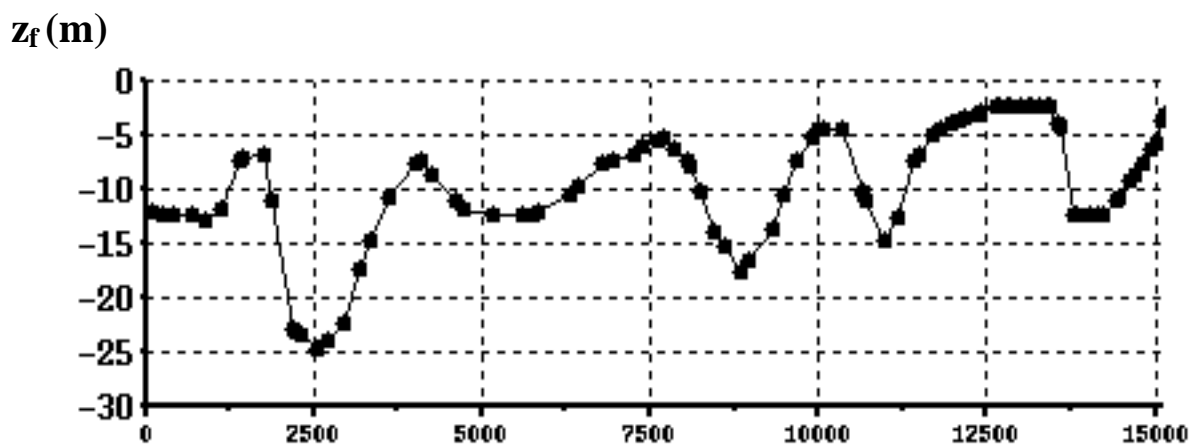

Figure 6a: Bathymétrie le long du profil

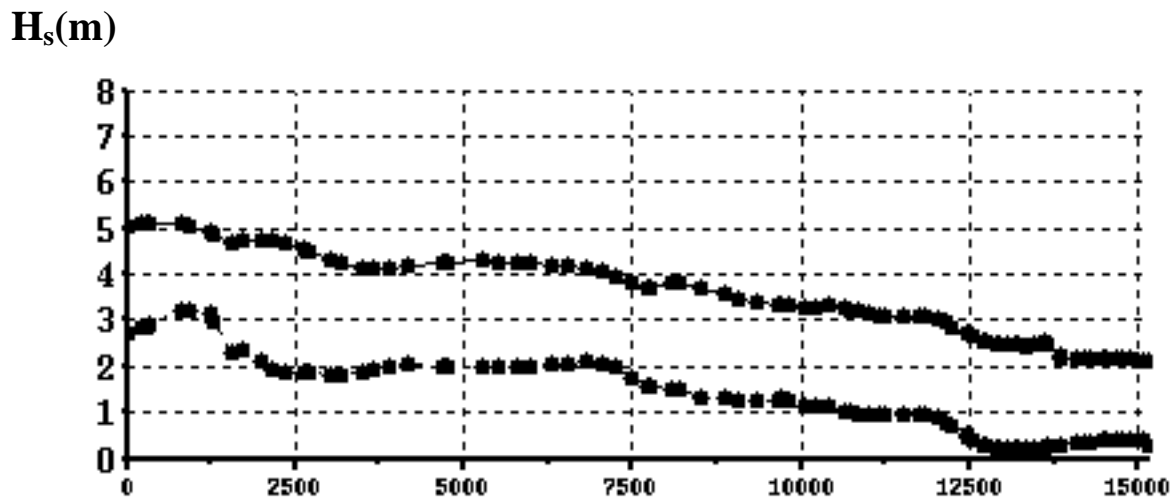

Figure 6b: Variation de la hauteur de houle (Tomawac : Trait plein : marée haute - Tirets : marée basse)

$\mathbf{Q}_{\mathrm{s}}\left(\mathbf{m}^{2} / \mathrm{s}\right)$

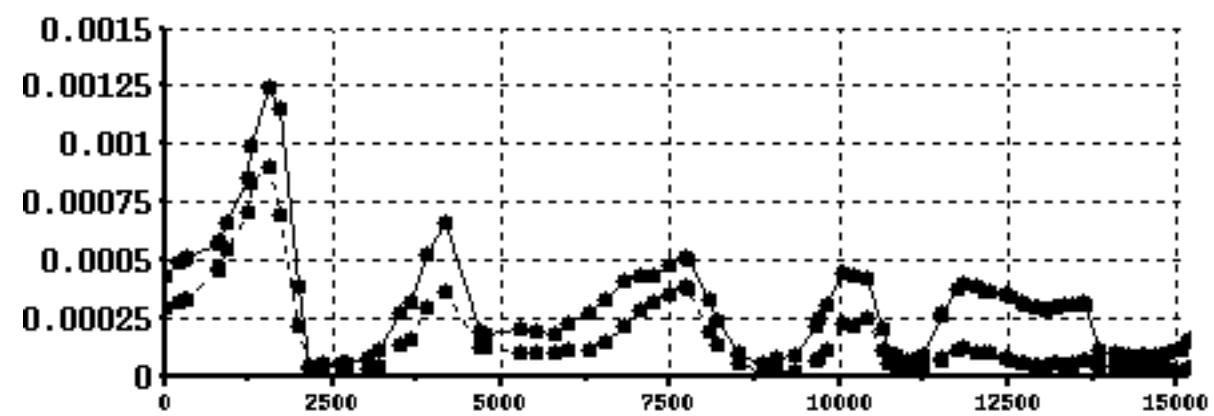

Figure 6c: Variation du taux de transport dans le profil de plage (Sisyphe : formule de Bijker ; $k_{s}={ }_{r}$ )

Figure 6: Résultats obtenus le long du profil de plage (Est de Dunkerque) 


\section{Conclusion}

Le transport littoral peut être calculé par une modélisation intégrée des forçages hydrodynamiques dus à la houle et au courant, puis du transport sédimentaire. L'effet de la houle est ici pris en compte par une augmentation des contraintes de frottement et par l'utilisation de formules de transport solide adaptées (instationnaires ou quasi-stationnaires). Le modèle utilisé tient compte aussi de la formation des rides par la houle et de leur influence sur le frottement.

La variabilité des résultats est importante, suivant les hypothèses retenues pour le calcul du frottement et le choix de la formule de transport. La précision des modèles de transport solide reste encore bien en-deçà de celle des calculs hydrodynamiques, en raison principalement de l'incertitude même des formules de transport solide et de leur méthodologie d'utilisation.

\section{Références}

1.Battjes J.A., Janssen J.P.F.M. ,1978 : Energy loss and set-up due to breaking of random waves. In Proc $16^{\text {th }}$ Int. Conf on Coast. Eng., ASCE, pp.569-587.

2.Bailard J. ,1981 : An energetic total load transport model for a plane sloping beach, Journal of Geophysical Research, 86, C11, 10938-10954.

3.Bijker E.W., 1992: Mechanics of sediment transport by the combination of waves and current, 23rd Int. Conf. on Coastal Engineering, 147-173.

4.Davies A.G, Villaret C., 2003: Modeling the effect of wave-induced ripples on littoral sand transport, Rapport EDF-LNH HP-75/2003/029.

5.Dibajnia M., Watanabe A., 1992: Sheet flow under non-linear waves and currents, Coast Eng., 2015-2029.

6.Hervouet J-M., 2003 : Hydrodynamique des écoulements à surface libre, modélisation numérique avec la méthode des éléments finis, Presses des Ponts et Chaussées.

7.Longuet-Higgins M.S., 1970 : The mechanics of the surf zone, Philos. Trans. R. Soc. London, (Cambridge UK).

8.Soulsby R.L., 1997: Dynamics of marine sands, HR Wallingford, T.Telford Publications.

9.Swart D.H., 1976 : Offshore sediment transport and equilibrium beach profiles. Delft Hydraulics Report, Publi. 131, Delft University.

10.Villaret C., 2001 Modélisation du transport solide par la formule de Bijker, Etude de sensibilité et tests validation, Rapport EDF-LNHE HP-75/2001/066.

11.Villaret C., 2003 : Intercomparaison des formules de transport solide, Programme biparti 2001, Rapport EDF-LNHE HP-75/2003/055.

12.Villaret C., 2004 : SISYPHE release v5.4 - User Manual, Rapport EDF-LNHE HP75/04/018.

13.Wiberg P.L., Harris C.K., 1994. Ripple geometry in wave-dominated environments, Journal of Geophysical Research 99 (C1), 775-789.

14. Shore Protection Manual, 1984, 4th ed., 1 Vol, U.S. Army Engineer Waterways Experiment Station, U.S. Govern. Printing Office, Washington DC.

15.Komar P.D., Inman, D.L.,1970 : Longshore Sand Transport on Beaches, Journal of Geophysical Research, Vol. 75, N³0, pp. 5914-5927. 